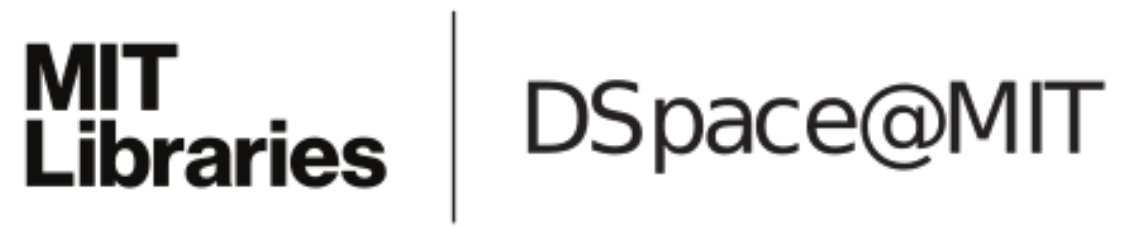

\author{
MIT Open Access Articles
}

Robustness of interdependent geometric networks under inhomogeneous failures

The MIT Faculty has made this article openly available. Please share how this access benefits you. Your story matters.

Citation: Kamran, Khashayar, Zhang, Jianan, Yeh, Edmund M and Modiano, Eytan H. 2018. "Robustness of interdependent geometric networks under inhomogeneous failures." 2018 16th International Symposium on Modeling and Optimization in Mobile, Ad Hoc, and Wireless Networks (WiOpt), 2018.

As Published: 10.23919/WIOPT.2018.8362877

Publisher: Institute of Electrical and Electronics Engineers (IEEE)

Persistent URL: https://hdl.handle.net/1721.1/126310

Version: Author's final manuscript: final author's manuscript post peer review, without publisher's formatting or copy editing

Terms of use: Creative Commons Attribution-Noncommercial-Share Alike 


\title{
Robustness of Interdependent Geometric Networks Under Inhomogeneous Failures
}

\author{
Khashayar Kamran*, Jianan Zhang ${ }^{\dagger}$, Edmund Yeh $^{\ddagger}$ and Eytan Modiano $§$ \\ ${ }^{*}$ Northeastern University, ${ }^{\dagger}$ Massachusetts Institute of Technology, \\ $\ddagger$ Northeastern University, ${ }^{\S}$ Massachusetts Institute of Technology, \\ Email: *kamrank@ece.neu.edu, †jianan@mit.edu, ${ }^{\ddagger}$ eyeh@ece.neu.edu, ${ }^{\S}$ modiano@mit.edu
}

\begin{abstract}
Complex systems such as smart cities and smart power grids rely heavily on their interdependent components. The failure of a component in one network may lead to the failure of the supported component in another network. Components which support a large number of interdependent components may be more vulnerable to attacks and failures. In this paper, we study the robustness of two interdependent networks under node failures. By modeling each network using a random geometric graph (RGG), we study conditions for the percolation of two interdependent RGGs after inhomogeneous node failures. We derive analytical bounds on the interdependent degree thresholds $\left(k_{1}, k_{2}\right)$, such that the interdependent RGGs percolate after removing nodes in $G_{i}$ that support more than $k_{j}$ nodes in $G_{j}$ $(\forall i, j \in\{1,2\}, i \neq j)$. We verify the bounds using numerical simulation, and show that there is a tradeoff between $k_{1}$ and $k_{2}$ for maintaining percolation after the failures.
\end{abstract}

\section{INTRODUCTION}

Large-scale networks are integral parts of complex cyber-physical systems and smart cities. Various entities from different networks rely on each other and boost each other's performance. For example, smart power grids depend on the designated communication network for effective control. At the same time, control centers and communication networks receive electrical power from the power grid to compute and transmit control signals. Failures in one network could have an impact on the other network, and could eventually lead to the failure of the whole system.

Cascading failures of interdependent networks under uniformly random node removals were first studied in [1]. Each network is modeled as a random graph. Nodes in one random graph depend on nodes in the other random graph, and a node is functional if both itself and its interdependent node are in the giant components of the their own graphs. After initial node failures in the first graph, their interdependent nodes in the second graph fail. Because of these failures, some nodes may be disconnected from the giant component of the second graph and then fail, and the failures of the disconnected nodes propagate back to nodes in the first graph.

Physical networks are spatially embedded. Nearby nodes are more likely to be connected, whereas distant nodes are less likely to be connected. Geographical proximity also applies to interdependence. In the Italy blackout in September 2003, where a cascading failure between power and communication networks took place, it is reported that every server in the communication network is connected to the geographically closest power station [1]. In [2], [3], an interdependent lattices model was proposed and the effect of removing nodes randomly or in a geographical region was analyzed. In [4], an interdependent Random Geometric Graphs (RGG) model was proposed. This model allows the two networks to have different sizes and average link lengths and also captures one-to-multiple dependencies with spatial constraints. The authors in [4] studied bounds on the percolation thresholds of interdependent RGGs. They studied the effect of random failures where every node fails independently with the same probability, and geographical failures where nodes are removed in a geographical region.

In real-world networks, failures may not be equally likely for every node. In a virus epidemic that infects smart grids, a power station with a large number of controllable communication nodes is more prone to infection and failure. Moreover, a power station that support a larger number of communication nodes is more likely to suffer physical attacks. Cyber attack is recognized as one of the major threats to the power grid [5], and the attack on a control center that controls a large number of power plants could have a more severe impact. In this paper, we study the robustness of interdependent RGGs under a failure model where the failure of each node depends on the number of its interdependent nodes. Our paper extends the analysis of degree-dependent failures in a single RGG in [6]. To the best of our knowledge, our paper is the first to address robustness of interdependent RGGs to nonuniform node failures in an analytical manner.

The main contributions of this paper are as follows.

1) We derive upper bounds on the interdependent degree thresholds $\left(k_{1}, k_{2}\right)$, such that the interdependent RGGs percolate after the removals of nodes in $G_{i}$ that are interdependent with more than $k_{j}$ nodes in $G_{j}(\forall i, j \in\{1,2\}, i \neq j)$.

2) We obtain a new closed-form upper bound on 
the degree threshold $k$, such that a single RGG remains percolated after the removals of nodes with more than $k$ neighbors. We then apply the result to obtain upper bounds on the interdependent degree thresholds $\left(k_{1}, k_{2}\right)$ for interdependent RGGs.

3) We verify the bounds using numerical simulation, and show that there is a trade-off between $k_{1}$ and $k_{2}$ for maintaining percolation after the failures.

\section{MODEL}

We study two interdependent Poisson boolean models $G_{\text {IntDep }}=\left(G_{1}\left(\lambda_{1}, d_{1}\right), G_{2}\left(\lambda_{2}, d_{2}\right), d_{\text {dep }}\right)$. In each graph $G_{i}\left(\lambda_{i}, d_{i}\right)$, nodes are generated by a Poisson point process of density $\lambda_{i}$ in an infinite twodimensional plane. Two nodes are connected if they are within distance $d_{i}(\forall i \in\{1,2\})$. Every node in $G_{i}$ is interdependent with all nodes in $G_{j}$ within distance $d_{\text {dep }}(\forall i, j \in\{1,2\}, i \neq j)$. Every node must have at least one interdependent node to be functional.

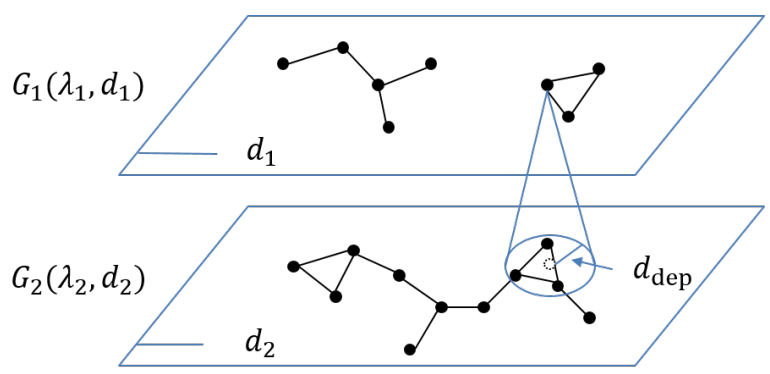

Fig. 1: Illustration of interdependent networks $G_{\text {IntDep }}=\left(G_{1}\left(\lambda_{1}, d_{1}\right), G_{2}\left(\lambda_{2}, d_{2}\right), d_{\text {dep }}\right)$.

Recall the definition of a mutual component and an infinite mutual component in interdependent graphs, as given in [4].

Definition 1. Let $V_{i}^{0}$ denote nodes in a connected component in $G_{i}\left(\lambda_{i}, d_{i}\right), \forall i \in\{1,2\}$. If each node in $V_{i} \subseteq V_{i}^{0}$ has at least one supply node in $V_{j} \subseteq V_{j}^{0}$ within $d_{\text {dep }}, \forall i, j \in\{1,2\}, i \neq j$, then nodes $V_{1}$ and $V_{2}$ form a mutual component of $G_{\text {IntDep }}$.

If, in addition, $V_{i}$ contains an infinite number of nodes, $\forall i \in\{1,2\}$, then $V_{1}$ and $V_{2}$ form an infinite mutual component.

The graph $G_{\text {IntDep }}$ percolates if there exists an infinite mutual component. The conditions for percolation have been studied in [4], where nodes in both graphs are generated by homogeneous Poisson point processes. In this paper, we study the percolation of $G_{\text {IntDep }}$ after removing nodes that have a large number of interdependent nodes. The removed nodes potentially have a significant impact on the percolation of $G_{\text {IntDep }}$, since they support a large number of nodes in the other graph. A power station that provides electrical power for a large number of routers, or a control center responsible for control of smart power stations in a large region, may both be vital in maintaining the robustness of the system.

The effect of removing such nodes cannot be viewed as a "thinning" of the original point process, since the remaining nodes do not follow Poisson point processes. This is therefore the main technical challenge in studying the percolation of $G_{\text {IntDep }}$, after the removal of all nodes with a large number of interdependent nodes. To address this issue, we develop mappings from Poisson point processes with inhomogeneous node removals to discrete models, and utilize the discrete models to derive conditions for the percolation of $G_{\text {IntDep }}$.

\section{Percolation After inhomogeneous NODE FAILURES}

In this section, we provide a sufficient condition for $G_{\text {IntDep }}$ to percolate, after removing all the nodes that are interdependent with a large number of nodes.

We first introduce a structure which is useful in our analysis. Consider a $(2 D-2 d) \times(D-2 d)$ rectangle $R(D)$, where $d=\max \left(d_{1}, d_{2}, d_{\mathrm{dep}}\right)$. If the mutual component in $G_{\text {IntDep }}$ forms a horizontal crossing $^{1}$ over $R(D)$, and a vertical crossing over the $(D-2 d) \times(D-2 d)$ square within the left half of $R(D)$, then the rectangle is complete. See Fig. 2 for an illustration.

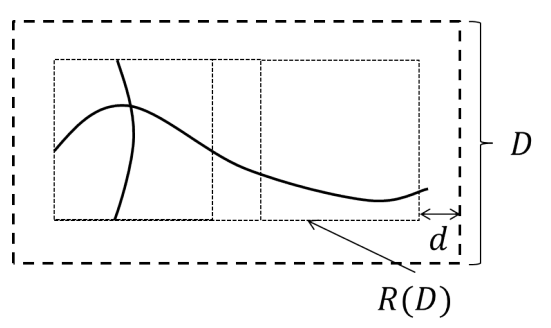

Fig. 2: Complete rectangle $R(D)$.

Consider two complete rectangles that have a sufficiently large overlapping area. The mutual component across one rectangle is connected to the mutual component across the other rectangle. Intuitively, if the probability that the rectangle is complete, denoted by $p_{c}[R(D)]$, is sufficiently large, then the mutual components across infinitely many overlapping rectangles could form an infinite mutual component. This method has been used to study the percolation of $G(\lambda, d)$ and $G_{\text {IntDep }}$ in [7], [4].

In this section, we study $G_{\text {IntDep }}$ which satisfies the condition that $p_{c}[R(D)]$ is greater than 0.8639 for

\footnotetext{
${ }^{1} \mathrm{~A}$ mutual component forms a horizontal crossing over a rectangle $\left[x_{1}, x_{2}\right] \times\left[y_{1}, y_{2}\right]$, if among the nodes in the mutual component, there exist a sequence of connected nodes in $G_{i}$, $\left(x_{i}^{1}, y_{i}^{1}\right), \ldots,\left(x_{i}^{m}, y_{i}^{m}\right)$, such that $x_{i}^{2}, \ldots, x_{i}^{m-1} \in\left[x_{1}, x_{2}\right]$, $x_{i}^{1}<x_{1}, x_{i}^{m}>x_{2}, y_{i}^{1}, \ldots, y_{i}^{m} \in\left[y_{1}, y_{2}\right], \forall i \in\{1,2\}$. A vertical crossing is defined analogously.
} 
some value $D$. For a single Poisson boolean model $G(\lambda, d)$, the probability $\tilde{p}_{c}[R(D)]$ that a rectangle is complete $^{2}$ approaches 1 as the size of the rectangle approaches infinity, as long as $G(\lambda, d)$ percolates [8]. Moreover, $\tilde{p}_{c}[R(D)]>0.8639$ for some $D$ is a sufficient condition for the percolation of $G(\lambda, d)$ [9]. Thus, the condition that $\tilde{p}_{c}[R(D)]>0.8639$ for some $D$ is equivalent to the condition that $G(\lambda, d)$ percolates.

The next theorem proves, under the assumption $p_{c}[R(D)]>0.8639$ for some value $D$, that $G_{\text {IntDep }}$ still contains an infinite mutual component after the removal of nodes that have a large number of interdependent nodes.

Theorem 1. If $p_{c}[R(D)]>0.8639$ for some $D$, then there exist $k_{1}, k_{2}$ such that $G_{\text {IntDep }}$ contains an infinite mutual component, after removing all the nodes in $G_{i}$ with more than $k_{j}$ interdependent nodes in $G_{j}, \forall i, j \in$ $\{1,2\}, i \neq j$.

Proof. We map the percolation of $G_{\text {IntDep }}$ to the percolation of a square lattice $L$. See Fig. 3 for an example. The state of a bond in $L$ is determined by the points in $G_{\text {IntDep }}$ in the rectangle containing the bond. A bond is open if the following conditions are satisfied.

1) Rectangle $R\left(3^{t} D\right)$ is complete.

2) There are no more than $k_{i}$ nodes from $G_{i}$ in a $\left(2 \times 3^{t} D\right) \times\left(3^{t} D\right)$ rectangle, $\forall i \in\{1,2\}$.

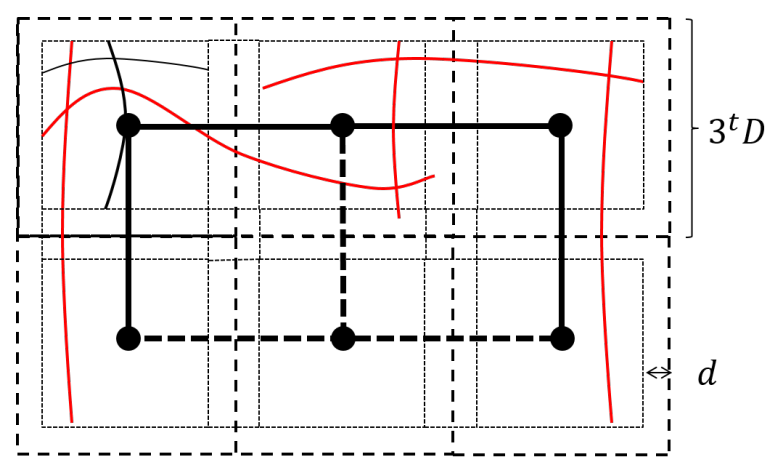

Fig. 3: 1-dependent bond percolation on square lattice $L$. Solid line segments of length $D$ represent open bonds, and dashed line segments represent closed bonds.

The event that a bond in $L$ is open is dependent on the event that an adjacent bond is open, since they both depend on the point processes of $G_{\text {IntDep }}$ in an overlapping area. However, the events that two nonadjacent bonds in $L$ are open are independent, since their associated rectangles do not overlap and the point processes in non-overlapping areas are independent. Thus, $L$ is a 1 -dependent bond percolation model on a square lattice. If the probability that a bond in $L$ is

\footnotetext{
${ }^{2}$ That is, a connected component in $G(\lambda, d)$ in the rectangle forms a horizontal and a vertical crossing.
}

open is larger than 0.8639 , then $L$ percolates [9]. This is in contrast with the independent bond percolation model where the percolation threshold is 0.5 [7].

The percolation of $L$ implies the percolation of $G_{\text {IntDep }}$. Consider two adjacent open bonds in $L$. The rectangles associated with the two bonds are both complete and the mutual components in both rectangles are joined by the nodes in the overlapping area of the two rectangles. For example, red curves across rectangles in Fig. 3 represent the mutual components in the rectangles, and they are joined if the open bonds are connected. If there is an infinite component in $L$, then there is an infinite mutual component in $G_{\text {IntDep }}$.

Moreover, none of the nodes adjacent to the open bonds are interdependent with more than $k_{i}$ nodes in $G_{i}, \forall i \in\{1,2\}$. For each open bond in $L$, the $(2 \times$ $\left.3^{t} D\right) \times\left(3^{t} D\right)$ rectangle contains at most $k_{i}$ nodes in $G_{i}$. Therefore, each node from $G_{i}$ in the $\left(2 \times 3^{t} D-\right.$ $2 d) \times\left(3^{t} D-2 d\right)$ rectangle $R$, where $d \geq d_{\mathrm{dep}}$, is interdependent with no more than $k_{j}$ nodes from $G_{j}$. To conclude, nodes in $G_{i}$ that have no more than $k_{j}$ interdependent nodes $\forall i, j \in\{1,2\}, i \neq j$ form an infinite mutual component.

Next, we compute the probability that a bond in $L$ is open. Recall that $p_{c}\left[R\left(3^{t} D\right)\right]$ is the probability that rectangle $R\left(3^{t} D\right)$ is complete. A result from [9] (proof of Theorem 2 therein) indicates that if $p_{c}[R(D)]>$ 0.8639 , then $p_{c}[R(3 D)]>p_{c}[R(D)]$, where $R(3 D)$ is a $(6 D-2 d) \times(3 D-2 d)$ rectangle. Thus, $p_{c}\left[R\left(3^{t} D\right)\right]$ is an increasing function in $t$, provided that $p_{c}[R(D)]>$ 0.8639 .

Since both $p_{c}\left[R\left(3^{t} D\right)\right]$ and $-1 /\left(2 \times 3^{2 t} D^{2} \lambda_{i}\right)$ are increasing functions in $t$, their sum is an increasing function in $t$. Let $t$ be the smallest integer such that

$p_{c}\left[R\left(3^{t} D\right)\right]-\frac{1}{2 \times 3^{2 t} D^{2} \lambda_{1}}-\frac{1}{2 \times 3^{2 t} D^{2} \lambda_{2}}>0.8639$.

Let $N_{i}^{t}$ denote the number of nodes from $G_{i}$ in a $\left(2 \times 3^{t} D\right) \times\left(3^{t} D\right)$ rectangle. The random variable $N_{i}^{t}$ follows a Poisson distribution with mean $\mathbb{E}\left[N_{i}^{t}\right]=2 \times$ $3^{2 t} D^{2} \lambda_{i}$ and variance $\operatorname{Var}\left[N_{i}^{t}\right]=2 \times 3^{2 t} D^{2} \lambda_{i}$. Let $k_{i}=4 \times 3^{2 t} D^{2} \lambda_{i}$. The probability that there are no more than $k_{i}$ nodes from $G_{i}$ in $R\left(3^{t} D\right)$ can be bounded using the Chebyshev's inequality.

$$
\begin{aligned}
\operatorname{Pr}\left(N_{i}^{t} \geq k_{i}\right) & \leq \operatorname{Pr}\left(\left|N_{i}^{t}-\mathbb{E}\left[N_{i}^{t}\right]\right| \geq \mathbb{E}\left[N_{i}^{t}\right]\right) \\
& \leq \frac{\operatorname{Var}\left[N_{i}^{t}\right]}{\left(\mathbb{E}\left[N_{i}^{t}\right]\right)^{2}}=\frac{1}{2 \times 3^{2 t} D^{2} \lambda_{i}} .
\end{aligned}
$$

The probability that either $N_{1}^{t} \geq k_{1}$ or $N_{2}^{t} \geq k_{2}$ can be bounded by the union bound

$\operatorname{Pr}\left(N_{1}^{t} \geq k_{1} \cup N_{2}^{t} \geq k_{2}\right) \leq \frac{1}{2 \times 3^{2 t} D^{2} \lambda_{1}}+\frac{1}{2 \times 3^{2 t} D^{2} \lambda_{2}}$

The probability that both $N_{1}^{t}<k_{1}$ and $N_{2}^{t}<k_{2}$ is at least $1-\operatorname{Pr}\left(N_{1}^{t} \geq k_{1} \cup N_{2}^{t} \geq k_{2}\right)$. 
For any two events $A$ and $B, \operatorname{Pr}(A \cap B) \geq \operatorname{Pr}(A)+$ $\operatorname{Pr}(B)-1$. Therefore, the probability that $R\left(3^{t} D\right)$ is complete and that there are no more than $k_{i}$ nodes from $G_{i}$ in $R\left(3^{t} D\right)$ is at least

$$
\begin{aligned}
& p_{c}\left[R\left(3^{t} D\right)\right]+\left(1-\frac{1}{2 \times 3^{2 t} D^{2} \lambda_{1}}-\frac{1}{2 \times 3^{2 t} D^{2} \lambda_{2}}\right) \\
& -1>0.8639 .
\end{aligned}
$$

Since the probability that a bond is open is at least $0.8639, L$ percolates. To conclude, $G_{\text {IntDep }}$ contains an infinite mutual component, in which every node in $G_{i}$ has no more than $k_{j}$ interdependent nodes in $G_{j}$, $\forall i, j \in\{1,2\}, i \neq j$.

\section{A NEW APPROACH FOR COMPUTING THE DEGREE THRESHOLD}

For the approach discussed in Section III, it is difficult to obtain an analytical expression for the probability $p_{c}\left[R\left(3^{t} D\right)\right]$ that a rectangle is complete. In this section, we develop a new approach that computes a closed-form solution to obtain degree thresholds. We first present the result for a single graph $G(\lambda, d)$ in Theorem 2, and then extend the result to $G_{\text {IntDep }}$ in Theorem 3.

Theorem 2. If there exist $\lambda, d, k_{t h}$ satisfying

$\sum_{k=8}^{k_{t h}} \frac{\left(\frac{98 d^{2} \lambda}{9}\right)^{k}}{k !} e^{-\frac{98 d^{2} \lambda}{9}} \max \left[1-8\left(\frac{97}{98}\right)^{k}, 0\right]>0.8639$,

then there exists an infinite component in $G(\lambda, d)$, after removing all the nodes with degree more than $k_{t h}$.

Proof. We map the percolation of $G(\lambda, d)$ to a 1dependent bond percolation model on a square lattice $L$. The state of a bond in $L$ is determined by the point process in a $2 D \times D$ rectangle $\hat{R}(D)$, where $D=7 d / 3$. See Fig. 4 for an illustration. A bond is open if the following conditions are satisfied.

1) There are no more than $k_{t h}$ nodes in $\hat{R}(D)$.

2) Each of the eight small squares contains at least one node.

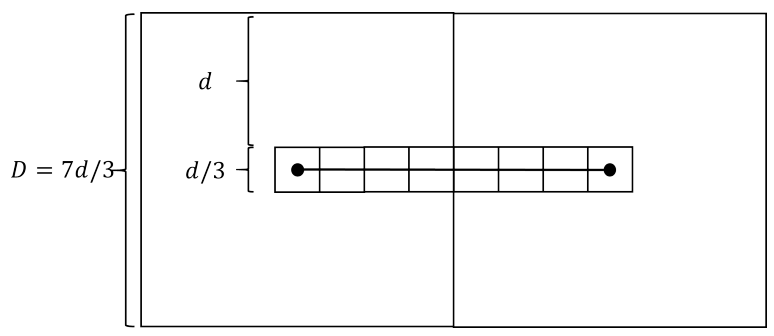

Fig. 4: A single bond of length $D$ and its associated $2 D \times D$ rectangle .

Notice that the rectangles $\hat{R}(D)$ associated with two non-adjacent bonds do not overlap, while $\hat{R}(D)$ associated with two adjacent bonds overlap. Therefore, the event that a bond is open is independent of the event that a non-adjacent bond is open, but is dependent on the event that an adjacent bond is open. Therefore, we study 1-dependent bond percolation in $L$.

We prove that the percolation of $L$ implies the percolation of $G(\lambda, d)$ after removing all nodes with degree larger than $k_{t h}$. If a bond is open in $L$, each small $d / 3 \times d / 3$ square contains at least one node in $G(\lambda, d)$. Nodes in two adjacent small squares are connected, because they are within distance $\sqrt{5} d / 3<d$. Moreover, all the nodes in the small squares have degrees no more than $k_{t h}$, because the total number of nodes in $\hat{R}(D)$ is no more than $k_{t h}$ and the distance between a node in the small squares and a node outside the rectangle $\hat{R}(D)$ is more than $d$. Next, consider two adjacent open bonds in $L$. The small squares that contain the intersection of the two bonds overlap. Thus, the nodes in the small squares adjacent to the two open bonds are connected. If the open bonds form an infinite component in $L$, then there is an infinite component in $G(\lambda, d)$ that contains only nodes with degrees no more than $k_{t h}$.

Next, we compute the probability that a bond is open. Let $S=2 D^{2}=98 d^{2} / 9$. The number of nodes in $\hat{R}(D)$ follows a Poisson distribution, i.e.,

$$
\operatorname{Pr}(N(S)=k)=\frac{(\lambda S)^{k}}{k !} e^{-\lambda S} .
$$

Given that a realization of the Poisson point process generates $k$ nodes in $\hat{R}(D)$, the $k$ nodes are located uniformly in $\hat{R}(D)$. The probability that there is no node in a small square of size $S^{\prime}=d / 3 \times d / 3$ within $\hat{R}(D)$ is

$$
\operatorname{Pr}\left(N\left(S^{\prime}\right)=0 \mid N(S)=k\right)=\left(\frac{S-S^{\prime}}{S}\right)^{k} .
$$

The probability that there exists at least one small square which contains no node, among a total of $h$ small squares, is upper bounded by the union bound

$$
h \operatorname{Pr}\left(N\left(S^{\prime}\right)=0 \mid N(S)=k\right)=h\left(\frac{S-S^{\prime}}{S}\right)^{k} .
$$

The probability that each of the $h$ small squares contains at least one node, given that the total number of nodes in $\hat{R}(D)$ is $k$, is at least

$$
\operatorname{Pr}(E \mid N(S)=k) \geq \max \left[1-h\left(\frac{S-S^{\prime}}{S}\right)^{k}, 0\right] .
$$

The probability that a bond is open is therefore

$$
\begin{aligned}
p & =\sum_{k=0}^{k_{t h}} \operatorname{Pr}(N(S)=k) \operatorname{Pr}(E \mid N(S)=k) \\
& \geq \sum_{k=8}^{k_{t h}} \frac{\left(98 d^{2} \lambda / 9\right)^{k}}{k !} e^{-98 d^{2} \lambda / 9} \max \left[1-8\left(\frac{97}{98}\right)^{k}, 0\right] .
\end{aligned}
$$


From [9], $p>0.8639$ is a sufficient condition for the percolation of $L$. Therefore, if $\lambda, d, k_{t h}$ satisfy $p>0.8639$, then $G(\lambda, d)$ percolates after removing all nodes with degree more than $k_{t h}$.

This result can be applied to interdependent networks to obtain a closed-form expression for the interdependent degrees $k_{1}$ and $k_{2}$ such that $G_{\text {IntDep }}$ percolates after removing all nodes which are interdependent with more than $k_{i}$ nodes in $G_{i}(\forall i \in\{1,2\})$. Theorem 3 provides a sufficient condition under which $G_{\text {IntDep }}$ percolates after removing nodes that are interdependent with a large number of nodes, under the condition $d_{1}=d_{2}=d_{\text {dep }}$.

Theorem 3. If there exist $\lambda_{i}, d, k_{i}$ satisfying

$\prod_{i=1}^{2} \sum_{k=8}^{k_{i}} \frac{\left(\frac{98 d^{2} \lambda_{i}}{9}\right)^{k}}{k !} e^{-\frac{98 d^{2} \lambda_{i}}{9}} \max \left[1-8\left(\frac{97}{98}\right)^{k}, 0\right]>0.8639$

then there exists an infinite mutual component in $G_{\text {IntDep }}=\left(G_{1}\left(\lambda_{1}, d\right), G_{2}\left(\lambda_{2}, d\right), d_{\text {dep }}=d\right)$, after removing all the nodes in $G_{i}$ that have more than $k_{j}$ interdependent nodes in $G_{j}(\forall i, j \in\{1,2\}, i \neq j)$.

Proof. The proof is similar to that for Theorem 2. In the construction of the 1-dependent bond percolation model $L$, a bond is open if

1) There are no more than $k_{i}$ nodes from $G_{i}$ in $\hat{R}(D)(\forall i \in\{1,2\})$.

2) Each of the eight small squares contains at least one node from $G_{i}(\forall i \in\{1,2\})$.

Given that the point processes in $G_{1}$ and $G_{2}$ are independent, the probability that a bond is open is the product of the probabilities that both conditions are satisfied for nodes in $G_{1}$ and $G_{2}$. From Theorem 2, the probability that nodes in $G_{i}$ satisfy both conditions is at least

$p_{i}=\sum_{k=8}^{k_{i}} \frac{\left(98 d^{2} \lambda_{i} / 9\right)^{k}}{k !} e^{-98 d^{2} \lambda_{i} / 9} \max \left[1-8\left(\frac{97}{98}\right)^{k}, 0\right]$.

If $p_{1} p_{2}>0.8639$, open bonds in $L$ form an infinite component.

Finally, we prove that the percolation of $L$ implies the percolation of $G_{\text {IntDep }}$ after removing nodes with a large number of interdependent nodes. By the same analysis as the proof of Theorem 2, and noting that nodes of $G_{1}$ and $G_{2}$ in the same small square are within distance $\sqrt{2} d / 3<d_{\text {dep }}$, nodes in $G_{\text {IntDep }}$ adjacent to the open bonds of $L$ form an infinite mutual component, and none of them has more than $k_{i}$ interdependent nodes from $G_{i}(\forall i \in\{1,2\})$.

\section{Simulation Results}

In this section, we first provide numerical results based on two finite-size interdependent RGGs in a $[0,20] \times[0,20]$ area. Nodes of the two RGGs are generated by Poisson point processes with densities

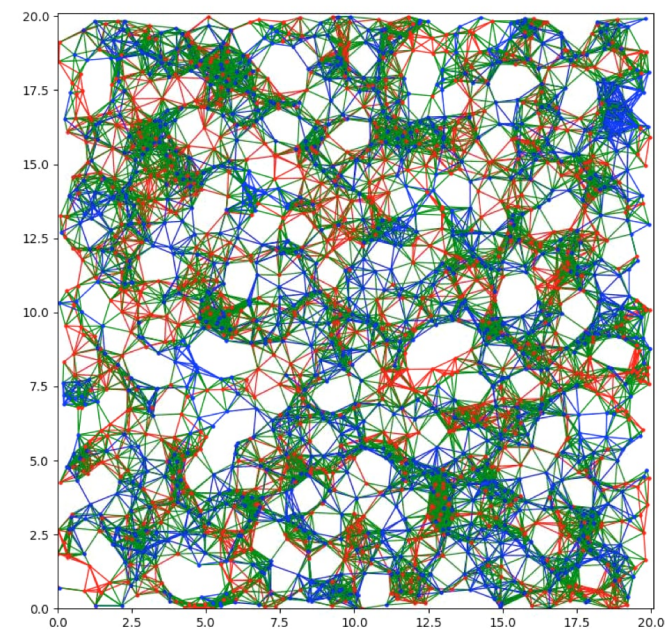

Fig. 5: Initial network with $\lambda_{1}=\lambda_{2}=2.5$ and $d_{1}=d_{2}=d_{d e p}$ before any failures. Red links and blue links belong to $G_{1}$ and $G_{2}$, respectively. Interdependent links are shown in green.

$\lambda_{1}=\lambda_{2}=2.5$. The connection distances and the interdependent distance are identical: $d_{1}=d_{2}=d_{\text {dep }}=1$. Before failures, the initial interdependent RGGs are percolated, as shown in Fig. 5.

After the removal of all nodes in $G_{1}$ with more than $k_{2}=8$ interdependent nodes in $G_{2}$ and all nodes in $G_{2}$ with more than $k_{1}=8$ interdependent nodes in $G_{1}$, the remaining graph is illustrated in Fig. 6a, where only 94 nodes remain out of the initial 2000 nodes and most of the network has lost its functionality. We repeat the same experiment with $k_{1}=k_{2}=10$ and as shown in Fig. 6b, 1475 nodes out of 2000 nodes have stayed functional. Based on the results of Theorems 1 and 3 , one can conclude the existence of a critical "threshold" for $k_{1}$ and $k_{2}$ for which removing nodes with more interdependent neighbors does not affect the robustness of network. To observe the transition, we set $k_{1}=k_{2}=k$ and plot the ratio of the size of the largest mutual component after failures to the size of the initial network as $k$ changes. The result is shown in Fig. 7.

Small values of $k_{1}$ and $k_{2}$ lead to more severe failures in the network, since a larger number of nodes are removed. There is a trade-off between the parameters $k_{1}$ and $k_{2}$ in the sense that if we increase one of them, small values of the other may not have the same devastating effect. To observe this trade-off, consider two finite interdependent RGG in a $[0,20] \times[0,20]$ area where nodes are generated according to Poison point processes with densities $\lambda_{1}=\lambda_{2}=5$ and $d_{1}=d_{2}=d_{\text {dep }}=1$. We have simulated the failures for a wide range of values for $k_{1}$ and $k_{2}$ and obtained the ratios of the size of the largest mutual component 


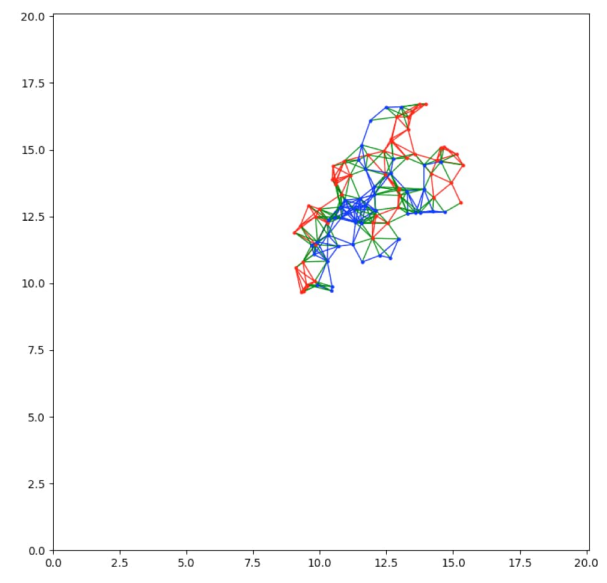

(a) $k=8$

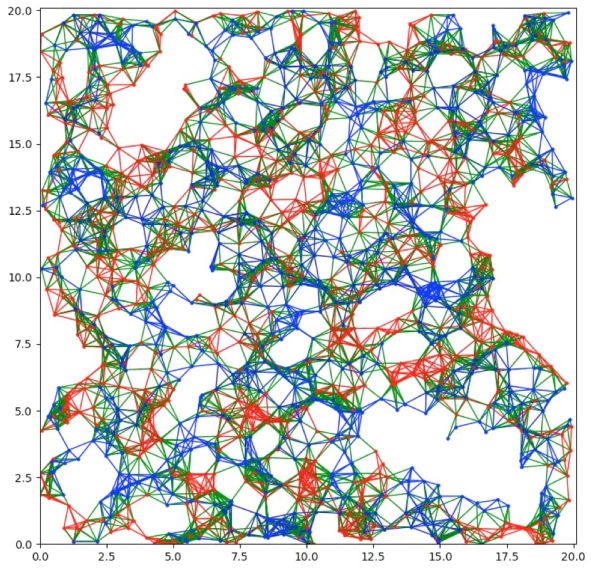

(b) $k=10$

Fig. 6: Resulting networks after removing nodes with interdependent degree more than $k$. Red links and blue links belong to $G_{1}$ and $G_{2}$, respectively. Interdependent links are shown in green.

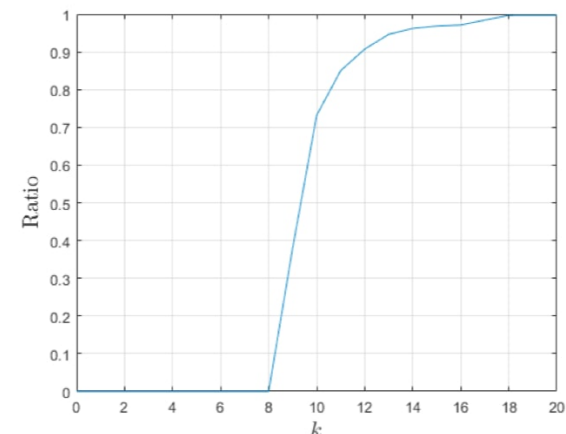

Fig. 7: Transition to critical region: $\lambda_{1}=\lambda_{2}=2.5$, $d_{1}=d_{2}=d_{d e p}=1$.

to the size of the network. The result is depicted in Fig. 8.

\section{CONCLUSION}

In this paper we analyzed the percolation of interdependent random geometric networks in an inhomogeneous failure scenario. We proved that even after removing all the nodes with more interdependent neighbors than a certain threshold, the network stays percolated. Using simulations, we demonstrated a trade-off between the interdependent degree thresholds: by increasing one, the network stays functional for smaller values of the other. The analysis of such failures can be used to consider more realistic cascading failure models where a node fails if a certain ratio of its interdependent neighbors fail.

\section{REFERENCES}

[1] S. V. Buldyrev, R. Parshani, G. Paul, H. E. Stanley, and S. Havlin, "Catastrophic cascade of failures in interdependent networks," Nature, vol. 464, no. 7291, pp. 1025-1028, 2010.

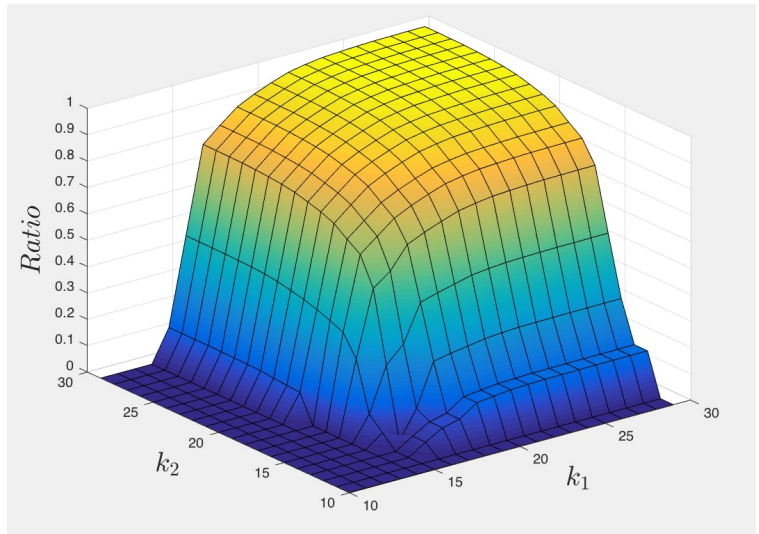

Fig. 8: Ratio of the size of the largest mutual component to the size of original network for different values of $k_{1}, k_{2}: \lambda_{1}=\lambda_{2}=5, d_{1}=d_{2}=d_{d e p}=1$.

[2] A. Bashan, Y. Berezin, S. V. Buldyrev, and S. Havlin, "The extreme vulnerability of interdependent spatially embedded networks," Nature Physics, vol. 9, no. 10, pp. 667-672, 2013.

[3] Y. Berezin, A. Bashan, M. M. Danziger, D. Li, and S. Havlin, "Localized attacks on spatially embedded networks with dependencies," Scientific reports, vol. 5, 2015.

[4] J. Zhang, E. Yeh, and E. Modiano, "Robustness of interdependent random geometric networks," in 2016 54th Annual Allerton Conference on Communication, Control, and Computing (Allerton). IEEE, 2016, pp. 172-179.

[5] National Academies of Sciences, Engineering, and Medicine, Enhancing the Resilience of the Nation's Electricity System. National Academies Press, 2017.

[6] Z. Kong and E. M. Yeh, "Resilience to degree-dependent and cascading node failures in random geometric networks," IEEE Trans. Inform. Theory, vol. 56, no. 11, pp. 5533-5546, 2010.

[7] B. Bollobás and O. Riordan, Percolation. Cambridge Univ. Press, 2006.

[8] R. Meester and R. Roy, Continuum percolation. Cambridge Univ. Press, 1996.

[9] P. Balister, B. Bollobás, and M. Walters, "Continuum percolation with steps in the square or the disc," Random Structures and Algorithms, vol. 26, no. 4, pp. 392-403, 2005. 